\title{
Novel prognostic nomograms for female patients with breast cancer and bone metastasis at presentation
}

\author{
Zhan Wang ${ }^{1}$, Yonggang Cheng ${ }^{1}$, Shi Chen ${ }^{1,2}$, Haiyu Shao ${ }^{1}$, Xiaowei Chen ${ }^{3}$, Zenan Wang ${ }^{1}$, Yucheng Wang ${ }^{4}$, \\ Hao Zhou ${ }^{1}$, Tao Chen ${ }^{1}$, Nong Lin ${ }^{1}$, Zhaoming Ye ${ }^{1}$ \\ ${ }^{1}$ Department of Orthopaedics, Centre for Orthopaedic Research, Orthopedics Research Institute of Zhejiang University, The Second Affiliated \\ Hospital, Zhejiang University School of Medicine, Hangzhou 310000, China; ${ }^{2}$ Department of Orthopaedics, Ninghai First Hospital, Ninghai \\ 315600, China; ${ }^{3}$ Department of Orthopaedics, Jingning Shezu Autonomous County People's Hospital, Lishui 323500, China; ${ }^{4}$ Graduate School of \\ Hebei North University, Zhangjiakou 075000, China \\ Contributions: (I) Conception and design: Z Ye, N Lin; (II) Administrative support: Z Ye; (III) Provision of study materials or patients: Z Wang, Y \\ Cheng, S Chen; (IV) Collection and assembly of data: H Shao, X Chen, Z Wang; (V) Data analysis and interpretation: Z Wang, Y Wang, H Zhou, T \\ Chen; (VI) Manuscript writing: All authors; (VII) Final approval of manuscript: All authors. \\ Correspondence to: Zhaoming Ye; Nong Lin. Department of Orthopaedics, Centre for Orthopaedic Research, Orthopedics Research Institute of \\ Zhejiang University, The Second Affiliated Hospital, Zhejiang University School of Medicine, 88 Jiefang Road, Hangzhou 310000 , China. \\ Email: yezhaoming@zju.edu.cn; linnong@zju.edu.cn.
}

Background: There is a paucity of literature about prognostic evaluation for patients with breast cancer (BC) and bone metastasis at presentation. To date, little is known about how to accurately predict the prognosis of BC patients with bone metastasis at presentation. Thus, an accurate prediction tool of prognosis in this population is urgently needed. Our goal is to construct novel and prognostic nomograms for BC patients with bone metastasis at presentation.

Methods: We searched Surveillance, Epidemiology, and End Results (SEER) database for BC patients with bone metastasis at presentation between 2010 and 2016. Multivariate analysis was performed to obtain significantly independent variables. Then, novel prognostic nomograms were constructed based on those independent predictors.

Results: Tumor grade, histological type, primary tumor size, tumor subtype, surgery, chemotherapy and number of metastatic organs except bone were recognized as significantly independent variables of both overall survival (OS) and cancer-specific survival (CSS). Then those significant variables were integrated to construct nomograms for 3-and 5-year survival. Calibration plots for the 3 - and 5-year survival in training and validation sets showed that the prediction curve was close to a 45 degree slash. The $\mathrm{C}$-indices of OS in training and validation cohorts were 0.705 and 0.678 , respectively. Similar results were observed for CSS in training and validation cohorts.

Conclusions: Our proposed nomograms can effectively and accurately predict the prognosis of BC patients with bone metastasis at presentation, which provide a basis for individual treatments for metastatic lesions.

Keywords: Breast cancer (BC); bone metastasis; nomogram; predictor; prognosis

Submitted Sep 12, 2019. Accepted for publication Dec 27, 2019.

doi: $10.21037 /$ atm.2020.01.37

View this article at: http://dx.doi.org/10.21037/atm.2020.01.37 


\section{Introduction}

Breast cancer (BC) is the most frequently diagnosed female malignance, which ranks the second leading cause of cancer death $(1,2)$. BC cells most frequently metastasize to the bone, with up to $75 \%$ of stage IV BC patients developing bone metastasis (3). Multiple systemic organ metastases from $\mathrm{BC}$ are common, and $17-37 \%$ patients have diseases limited to the skeleton (4-6). Patients with bone-only first metastasis tend to experience a better prognosis than those with otheronly first metastasis (7). Patients with disease that remains confined to the bone have longer survival than patients with subsequent visceral involvement (8). Bone metastasis can result in poor survival, considerable morbidity, intractable pain and decreased quality of life (9-11).

The 3- and 5-year survival rates of $\mathrm{BC}$ patients with bone metastasis were $25 \%$ and $13 \%$, respectively (12). Pogoda et al. (13) reported that the median overall survival (OS) was only 5.5 months after the detection of bone metastasis among triple-negative $\mathrm{BC}$ patients. However, with the development of hormone or bone-targeted drug therapies, BC patients including metastatic BC patients experienced a better prognosis $(14,15)$. Additionally, surgery or radiotherapy for patients with bone metastasis can provide effective local control and improve quality of life (16-20), especially for patients with pathologic fractures (9).

To our knowledge, the risk factors and their effects on prognosis of patients with $\mathrm{BC}$ and bone metastasis are rarely explored. Ahn et al. (21) reported that bisphosphonate treatment was the most significant positive predictor of OS among BC patients with bone-only metastasis. Amanda Parkes et al. (22) found that multiple bone metastasis and both axial and appendicular skeleton involvement were independent predictors of decreased OS. Among BC patients with spine metastases, Zhao et al. (23) found that no visceral metastasis, solitary spine metastasis and postoperative chemotherapy performed were independent prognostic factors of increased OS. Other significant independent predictors for survival among patients with $\mathrm{BC}$ and bone metastasis were race, age, tumor grade, tumor subtype, surgery for primary tumor (24).

Standard treatments for patients with BC and bone metastasis are lacking. In order to provide personalized and reasonable treatment strategies, we need to make an accurate prediction of outcome in patients with $\mathrm{BC}$ and bone metastasis. Recently, the nomogram is widely used in various cancers to conveniently and accurately predict the outcomes (25-27). It can be recognized as a helpful tool in terms of multidisciplinary decision-making and optimizing treatment options especially for metastatic lesions. However, no systematic attempts have ever been made to develop prognostic nomograms for BC patients with bone metastasis. Therefore, we aim to develop and validate nomograms for those patients and assist clinicians to accurately predict survival.

\section{Methods}

\section{Patients selection and data acquisition}

From 2010 to 2016, patients with a diagnosis of BC and bone metastasis at presentation were identified using the Surveillance, Epidemiology, and End Results (SEER) database (https://seer.cancer.gov/). Patient data extraction were performed using the case-listing session procedure from the SEER program (28).

Variables selected from SEER database were as follows: race, age, gender, laterality, pathological pattern, tumor grade, T, N stage (AJCC stage group 7th edition, 2010), tumor size, tumor subtype, surgery, radiotherapy, chemotherapy, cause of death, vital status and survival time. Patients were included according to the following criteria: (I) female patients; (II) diagnosis confirmed by histology; (III) age at diagnosis $20-80$ years. Patients were excluded for the following reasons: (I) cases with a diagnosis according to clinical or imaging findings or autopsy; (II) cases with unknown variables; (III) cases with unknown survival time or survival time less than one month (Figure 1).

A total of 5,860 patients with $\mathrm{BC}$ and bone metastasis at presentation were identified from 18 SEER registries. We randomly selected patients from nine registries (Detroit, Alaska Natives, Atlanta, Kentucky, Greater Hawaii, Iowa, Georgia, Connecticut, and California) as the training cohort $(n=3,311)$, and patients from the other nine registries were regarded as the validation cohort $(n=2,549)$. Radiotherapy or surgical treatment in this research was performed to treat the primary lesion.

\section{Statistical analysis}

We first performed univariate and multivariate Cox regression analysis to evaluate and reveal significant risk factors of OS or cancer-specific survival (CSS). CSS was calculated from initial diagnosis to death specific to the cancer-related diagnosis (28). Meanwhile, we obtained the hazard ratios (HRs) with $95 \%$ confidence intervals (CIs) of 


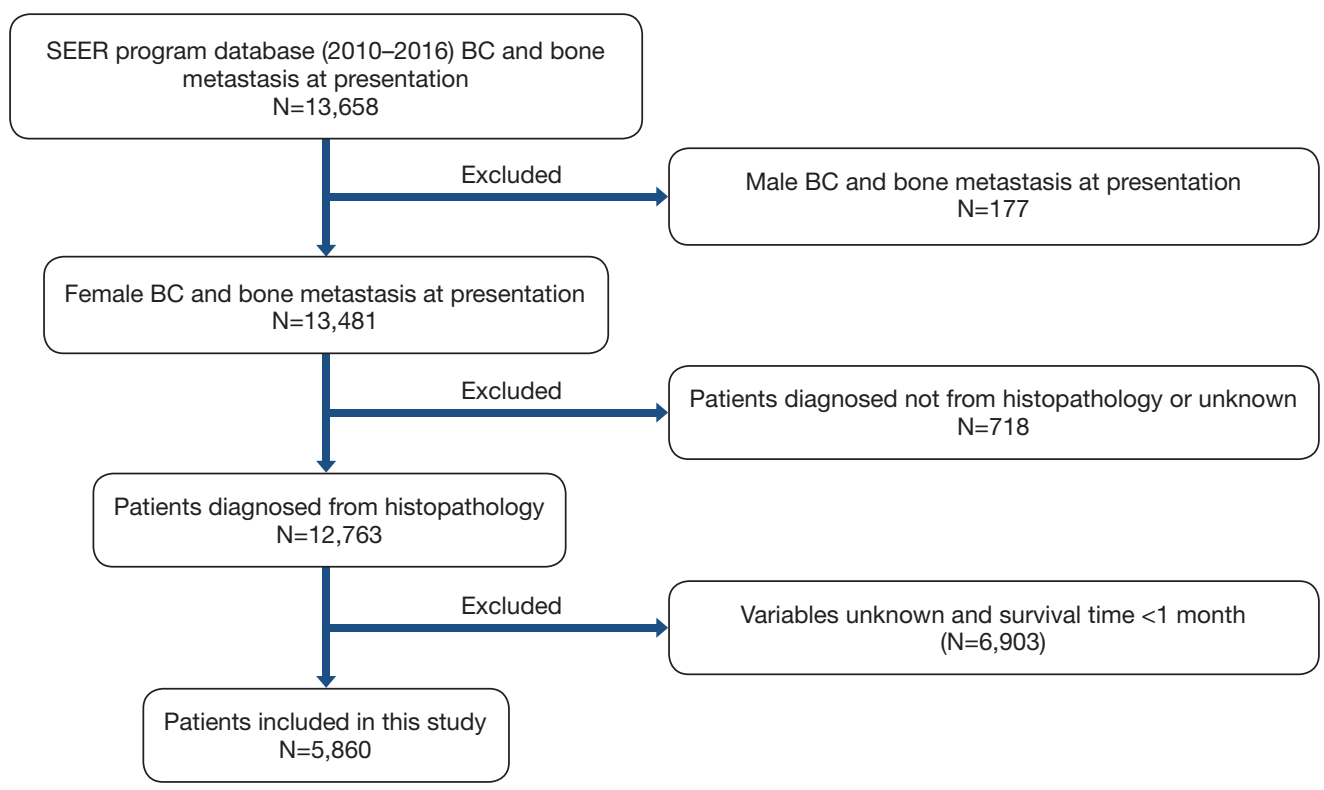

Figure 1 The flow chart for selection of study population.

various predictors. IBM SPSS Statistics v220.0 was used for the above statistical analyses.

Nomograms were constructed and validated based on a set of significant variables of multivariate analysis from the training set. The performance of prognostic models was evaluated based on concordance index (C-index) and calibration plots in training and validation cohorts (29). We performed bootstraps with 1,000 resamples to validate the nomograms in training and validation cohorts (30). $\mathrm{R}$ version 3.6.0 software (https://www.r-project.org/) was applied for the above statistical analyses.

\section{Results}

\section{Baseline characteristics}

Detailed clinical characteristics of all cases were shown in Table 1. All BC patients had bone metastasis at initial diagnosis. Mean and median ages of the entire cohort were 57 and 58 years (range, 21-80 years), respectively. More than three out of four patients $(n=4,455,76.0 \%)$ were white race. About half $(n=3,021,51.6 \%)$ of the patients were leftorigin of primary BC. Majority of patients were diagnosed with tumor grade II or III/IV BC (46.7\% and $44.1 \%$, respectively), and only 535 patients $(9.1 \%)$ were with tumor grade I. The most common histological type was ductal cancer $(78.5 \%)$. The mean and median tumor sizes were
5.1 and $4.1 \mathrm{~cm}$, respectively. The proportions of patients diagnosed with luminal A, luminal B, HER2 ${ }^{+}$and triplenegative were $65.5 \%, 18.2 \%, 6.7 \%$ and $9.6 \%$, respectively. Almost half of all patients received radiotherapy $(n=2,483$, $42.4 \%)$. About one third of patients had surgical treatment $(\mathrm{n}=2,131,36.4 \%)$. Approximately two-thirds of patients received chemotherapy $(n=3,650,62.3 \%)$. In terms of metastatic types, 3,520 patients $(60.1 \%)$ had bone-only metastatic pattern, 1,684 patients $(28.7 \%)$ had bone and one visceral metastatic pattern, 656 patients $(11.2 \%)$ had bone and $\geq 2$ visceral metastatic pattern. The survival outcome was poor with 5 -year OS and CSS rate of $31.4 \%$ and $33.8 \%$, respectively.

\section{Independent predictors in the study population}

The detailed results of the univariate analysis from the training set were shown in Table 2. Tumor grade, T stage, histological type, tumor size, tumor subtype, surgery, chemotherapy and number of metastatic organs except bone were shown to be significantly associated with both OS and CSS. Then those significant variables were included for further multivariate Cox proportional-hazard regression analysis. We used the backwards selection procedure to perform the variable selection, which is helpful for determine the independent predictors that effectively contribute to patients' survival. Ultimately, tumor grade, 
Table 1 Clinical characteristics of 5,860 patients with breast cancer with identified bone metastases at diagnosis

\begin{tabular}{|c|c|}
\hline Variable & Value \\
\hline \multicolumn{2}{|l|}{ Age (years) } \\
\hline Mean & 57 \\
\hline Median & 58 \\
\hline $20-40$ & $614(10.5)$ \\
\hline $41-60$ & 2,755 (47.0) \\
\hline $61-80$ & $2,491(42.5)$ \\
\hline \multicolumn{2}{|l|}{ Race } \\
\hline White & 4,455 (76.0) \\
\hline Black & $926(15.8)$ \\
\hline Others & 479 (8.2) \\
\hline \multicolumn{2}{|l|}{ Laterality } \\
\hline Left & $3,021(51.6)$ \\
\hline Right & $2,839(48.4)$ \\
\hline \multicolumn{2}{|l|}{ Tumor grade } \\
\hline I & $535(9.1)$ \\
\hline ॥ & $2,739(46.7)$ \\
\hline III/IV & $2,586(44.1)$ \\
\hline \multicolumn{2}{|l|}{ T stage } \\
\hline $\mathrm{T} 1$ & $752(12.8)$ \\
\hline $\mathrm{T} 2$ & $2,182(37.2)$ \\
\hline T3 & $1,188(20.3)$ \\
\hline $\mathrm{T} 4$ & $1,738(29.7)$ \\
\hline \multicolumn{2}{|l|}{$\mathrm{N}$ stage } \\
\hline No & $1,253(21.4)$ \\
\hline $\mathrm{N} 1$ & $2,840(48.5)$ \\
\hline $\mathrm{N} 2$ & $803(13.7)$ \\
\hline N3 & $964(16.5)$ \\
\hline \multicolumn{2}{|l|}{ Histological type } \\
\hline Ductal & $4,603(78.5)$ \\
\hline Lobular & $657(11.2)$ \\
\hline Mixed ductal and lobular & $328(5.6)$ \\
\hline Others & $272(4.6)$ \\
\hline \multicolumn{2}{|l|}{ Tumor size (cm) } \\
\hline Mean & 5.1 \\
\hline Median & 4.1 \\
\hline$<5$ & 3,417 (58.3) \\
\hline
\end{tabular}

Table 1 (continued)
Table 1 (continued)

\begin{tabular}{cc}
\hline Variable & Value \\
\hline $5-10$ & $2,062(35.2)$ \\
$>10$ & $381(6.5)$
\end{tabular}

Tumor subtype

Luminal A

$3,839(65.5)$

Luminal B

1,065 (18.2)

HER2+

$391(6.7)$

Triple-negative

$565(9.6)$

Surgery

Yes

$2,131(36.4)$

No

$3,729(63.6)$

Radiotherapy

Yes

2,483 (42.4)

No

$3,377(57.6)$

Chemotherapy

Yes

$3,650(62.3)$

No

$2,210(37.7)$

Brain metastasis

Yes

$360(6.1)$

No

$5,500(93.9)$

Liver metastasis

Yes

1,331 (22.7)

No

$4,529(77.3)$

Lung metastasis

Yes

$1,396(23.8)$

No

$4,464(76.2)$

Number of metastatic organs except bone

0

$3,520(60.1)$

1

$\geq 2$

1,684 (28.7)

$656(11.2)$

Status

Alive

$3,086(52.7)$

Dead

2,774 (47.3)

3-year OS rate

$51.7 \%$

3-year CSS rate

$53.6 \%$

5-year OS rate

$31.4 \%$

5-year CSS rate

$33.8 \%$

Grade I: well differentiated; Grade II: moderately differentiated; Grade III: poorly differentiated; Grade IV: undifferentiated anaplastic. OS, overall survival; CSS, cancer-specific survival. 
Table 2 Univariate Cox regression analysis of OS and CSS in the training cohort

\begin{tabular}{|c|c|c|c|c|c|c|}
\hline Variable & \multicolumn{3}{|c|}{ OS } & \multicolumn{3}{|c|}{ cSS } \\
\hline \multicolumn{7}{|l|}{ Age (years) } \\
\hline $20-40$ & 1 & & & 1 & & \\
\hline $41-60$ & 0.922 & $0.779-1.092$ & 0.347 & 0.945 & $0.790-1.130$ & 0.534 \\
\hline \multicolumn{7}{|l|}{ Race } \\
\hline White & 1 & & & 1 & & \\
\hline Black & 1.076 & $0.941-1.231$ & 0.282 & 1.061 & $0.921-1.222$ & 0.413 \\
\hline Others & 1.114 & $0.934-1.329$ & 0.229 & 1.075 & $0.891-1.298$ & 0.447 \\
\hline Right & 1.043 & $0.946-1.150$ & 0.397 & 1.04 & $0.939-1.152$ & 0.454 \\
\hline \multicolumn{7}{|l|}{ Tumor grade } \\
\hline I & 1 & & & 1 & & \\
\hline II & 1.299 & $1.065-1.585$ & 0.01 & 1.404 & $1.130-1.745$ & 0.002 \\
\hline III/IV & 1.952 & $1.605-2.375$ & $<0.001$ & 2.155 & $1.739-2.670$ & $<0.001$ \\
\hline \multicolumn{7}{|l|}{ T stage } \\
\hline $\mathrm{T} 1$ & 1 & & & 1 & & \\
\hline $\mathrm{T} 2$ & 1.002 & $0.847-1.187$ & 0.978 & 0.97 & $0.813-1.157$ & 0.733 \\
\hline \multicolumn{7}{|l|}{$\mathrm{N}$ stage } \\
\hline N1 & 0.99 & $0.872-1.124$ & 0.878 & 0.998 & $0.872-1.141$ & 0.972 \\
\hline N2 & 0.902 & $0.762-1.069$ & 0.234 & 0.899 & $0.751-1.077$ & 0.248 \\
\hline N3 & 1.003 & $0.858-1.173$ & 0.967 & 1.042 & $0.884-1.227$ & 0.627 \\
\hline \multicolumn{7}{|l|}{ Histological type } \\
\hline Ductal & 1 & & & 1 & & \\
\hline Lobular & 0.959 & $0.817-1.125$ & 0.606 & 0.964 & $0.815-1.140$ & 0.67 \\
\hline Mixed ductal and lobular & 0.857 & $0.684-1.074$ & 0.181 & 0.859 & $0.678-1.088$ & 0.208 \\
\hline Others & 1.642 & $1.349-1.999$ & $<0.001$ & 1.705 & $1.392-2.088$ & $<0.001$ \\
\hline \multicolumn{7}{|l|}{ Tumor size $(\mathrm{cm})$} \\
\hline$<5$ & 1 & & & 1 & & \\
\hline $5-10$ & 1.235 & $1.114-1.369$ & $<0.001$ & 1.263 & $1.134-1.408$ & $<0.001$ \\
\hline$>10$ & 1.836 & $1.521-2.215$ & $<0.001$ & 1.954 & $1.613-2.368$ & $<0.001$ \\
\hline
\end{tabular}

Table 2 (continued) 
Table 2 (continued)

\begin{tabular}{|c|c|c|c|c|c|c|}
\hline Variable & \multicolumn{3}{|c|}{ OS } & \multicolumn{3}{|c|}{ CSS } \\
\hline \multicolumn{7}{|l|}{ Tumor subtype } \\
\hline Luminal A & 1 & & & 1 & & \\
\hline Luminal B & 0.883 & $0.767-1.017$ & 0.085 & 0.865 & $0.746-1.004$ & 0.056 \\
\hline Triple-negative & 3.366 & $2.931-3.866$ & $<0.001$ & 3.482 & $3.013-4.023$ & $<0.001$ \\
\hline \multicolumn{7}{|l|}{ Surgery } \\
\hline Yes & 1 & & & 1 & & \\
\hline No & 1.842 & $1.658-2.047$ & $<0.001$ & 1.814 & $1.624-2.026$ & $<0.001$ \\
\hline No & 0.996 & $0.903-1.098$ & 0.928 & 0.959 & $0.865-1.063$ & 0.424 \\
\hline \multicolumn{7}{|l|}{ Chemotherapy } \\
\hline Yes & 1 & & & 1 & & \\
\hline No & 1.269 & $1.150-1.401$ & $<0.001$ & 1.238 & $1.116-1.374$ & $<0.001$ \\
\hline \multicolumn{7}{|c|}{$\begin{array}{l}\text { Number of metastatic organs } \\
\text { except bone }\end{array}$} \\
\hline 0 & 1 & & & 1 & & \\
\hline 1 & 1.678 & $1.504-1.873$ & $<0.001$ & 1.735 & $1.547-1.947$ & $<0.001$ \\
\hline
\end{tabular}

Grade I: well differentiated; Grade II: moderately differentiated; Grade III: poorly differentiated; Grade IV: undifferentiated anaplastic. OS, overall survival; CSS, cancer-specific survival.

histological type, tumor size, tumor subtype, surgery, chemotherapy and number of metastatic organs except bone were included to develop the nomogram. Multivariate analysis of the training set also revealed that these final seven variables were significant predictors of OS and CSS (Table 3).

\section{Prognostic nomogram building and validation}

For the development of nomogram for BC patients with bone metastasis, final seven independent risk factors of survival from the training set were incorporated. The nomograms (Figures 2,3) revealed that tumor subtype and number of metastatic organs except bone contributed most to both OS and CSS. Nomogram as a predictive tool is quite user-friendly. Clinicians or patients can sum the scores of each covariate and draw a vertical line straight downwards to determine the probabilities of survival of each patient. The scores assigned to each variable can be viewed in detail in Table 4.

For instance, a BC woman patient presented bone metastasis at diagnosis. The primary tumor was identified as grade II and ductal type with size $6.0 \mathrm{~cm}$. The tumor subtype proved to be luminal B. She then received surgery for primary tumor and chemotherapy. No other visceral metastasis was found. We add the scores of all the variables together to get the total scores of 8.2 points for OS and 8.8 points for CSS. Thus, this patient's corresponding 3-year OS and cancer specific survival rates were about $70 \%$ and $72 \%$, respectively.

Performance of models was validated and revealed internally and externally. As shown in Figures 4 and 5, the prediction curves in both training and validation sets were close to a 45 degree slash, which indicated that 
Table 3 Multivariate Cox regression analysis of OS and CSS in the training cohort

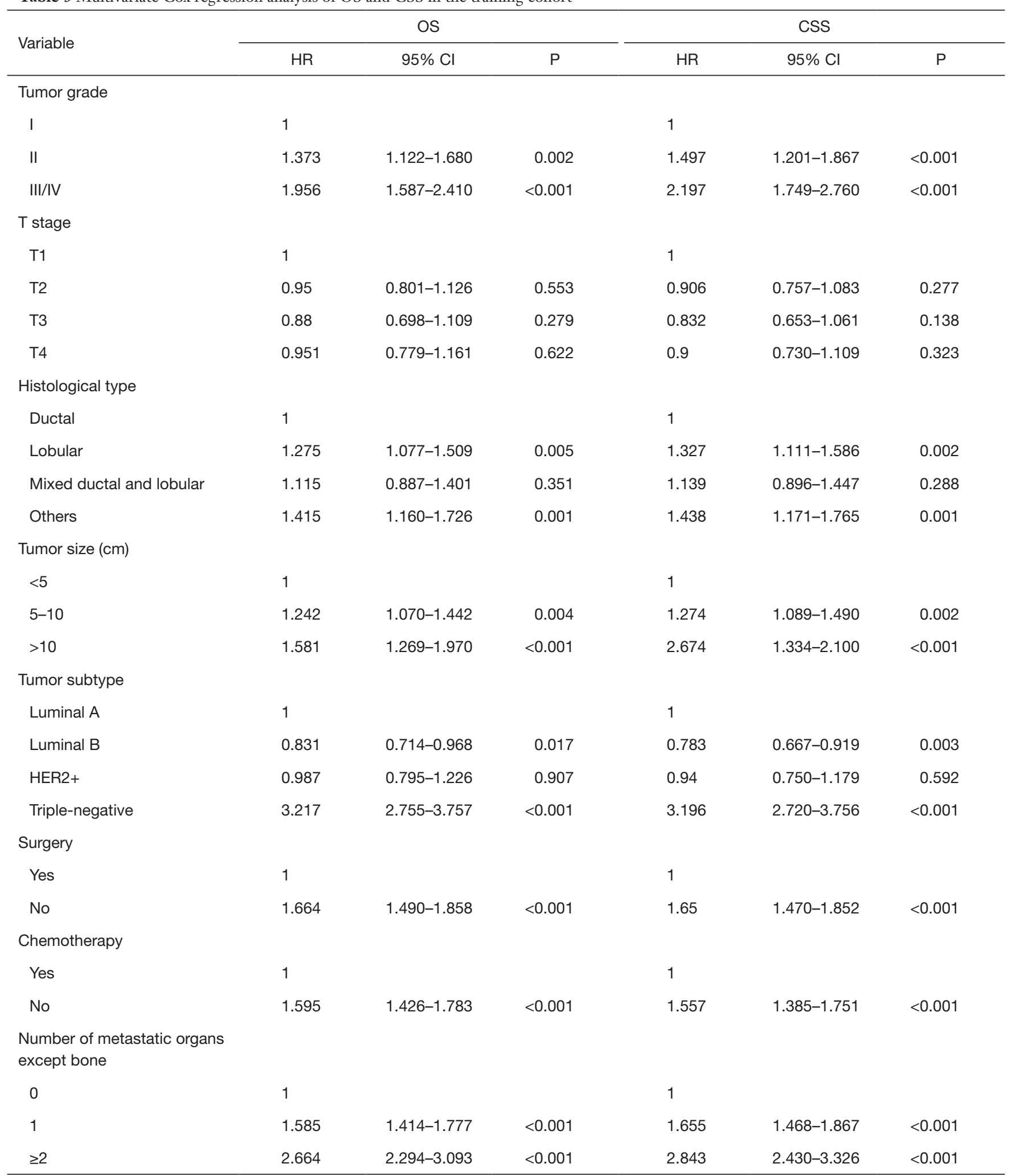

Grade I: well differentiated; Grade II: moderately differentiated; Grade III: poorly differentiated; Grade IV: undifferentiated anaplastic. OS, overall survival; CSS, cancer-specific survival. 


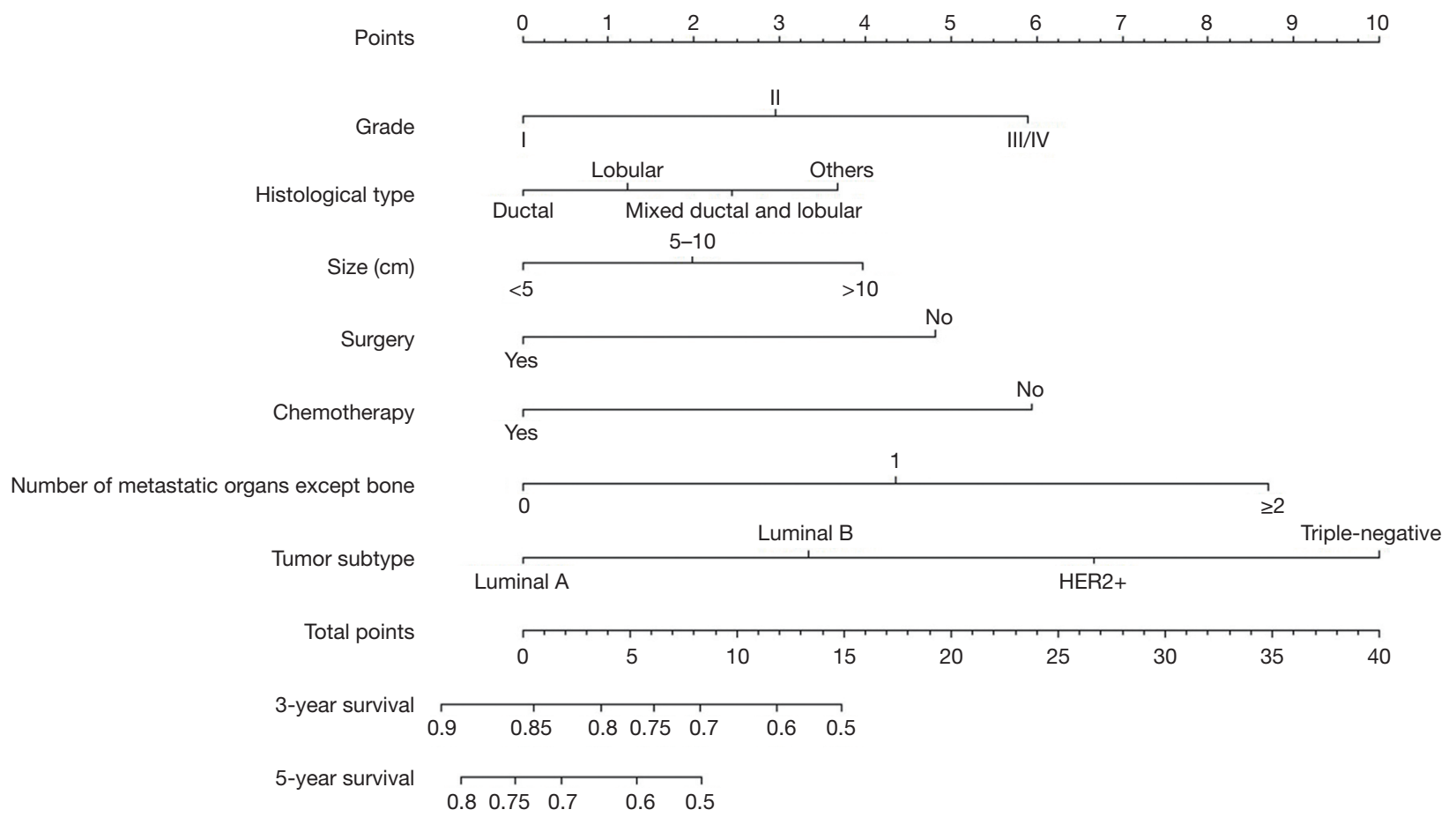

Figure 2 Nomogram for predicting 3-and 5-year overall survival of patients with breast cancer and bone metastasis at presentation.

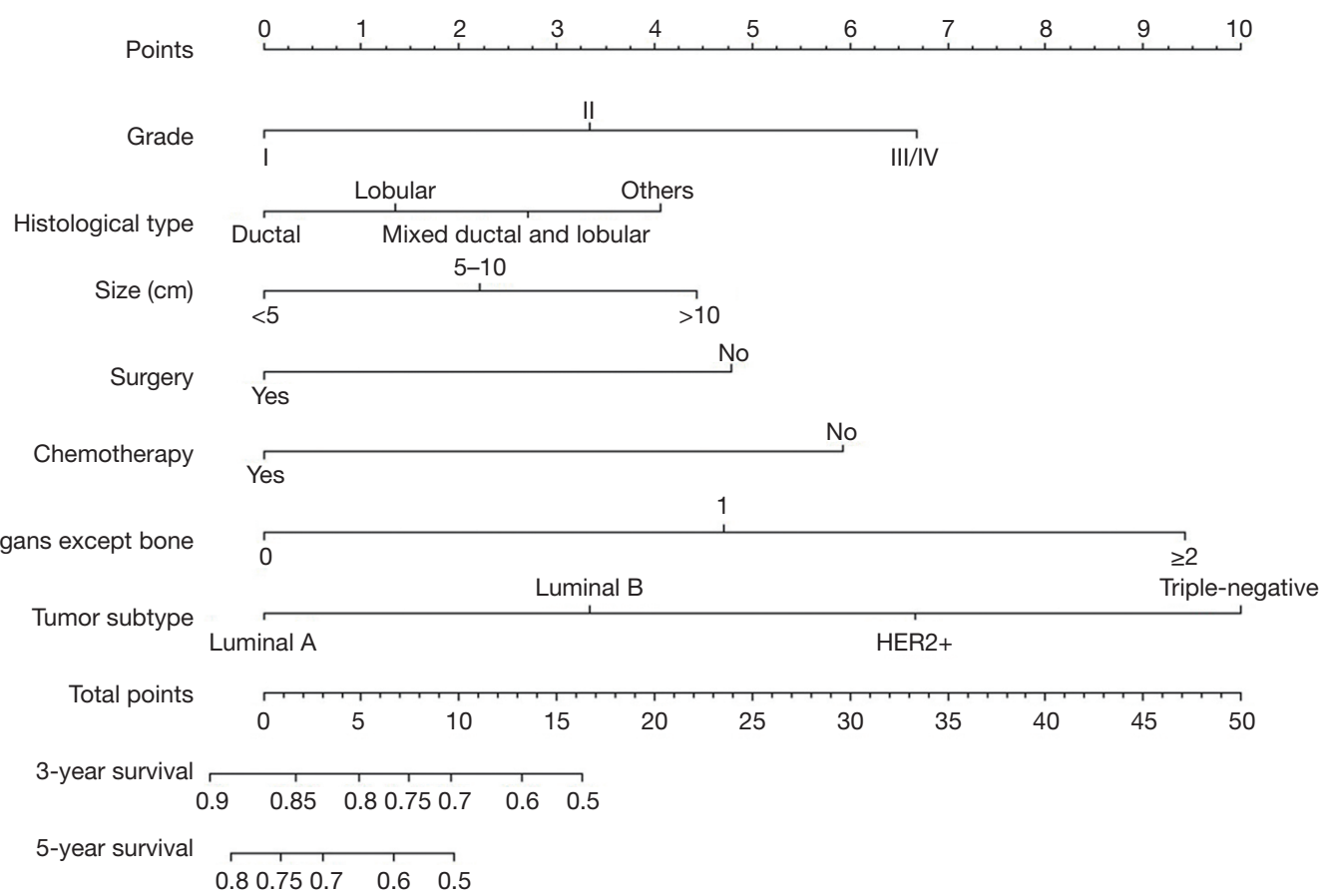

Figure 3 Nomogram predicting 3- and 5-year cancer-specific survival of patients with breast cancer and bone metastasis at presentation. 
Table 4 Point assignment for specific categories of the variables included in the nomograms

\begin{tabular}{|c|c|c|}
\hline Variable & OS nomogram & CSS nomogram \\
\hline \multicolumn{3}{|l|}{ Tumor grade } \\
\hline I & 0 & 0 \\
\hline II & 2.9 & 3.3 \\
\hline III/IV & 5.9 & 6.7 \\
\hline \multicolumn{3}{|l|}{ Histological types } \\
\hline Ductal & 0 & 0 \\
\hline Lobular & 1.2 & 1.4 \\
\hline $\begin{array}{l}\text { Mixed ductal and } \\
\text { lobular }\end{array}$ & 2.4 & 2.7 \\
\hline Others & 3.7 & 4.1 \\
\hline \multicolumn{3}{|l|}{ Tumor size $(\mathrm{cm})$} \\
\hline$<5$ & 0 & 0 \\
\hline $5-10$ & 2 & 2.2 \\
\hline$>10$ & 4 & 4.4 \\
\hline \multicolumn{3}{|l|}{ Tumor subtype } \\
\hline Luminal A & 0 & 0 \\
\hline Luminal B & 3.3 & 3.3 \\
\hline HER2+ & 6.7 & 6.7 \\
\hline Triple-negative & 10 & 10 \\
\hline \multicolumn{3}{|l|}{ Surgery } \\
\hline Yes & 0 & 0 \\
\hline No & 4.8 & 4.8 \\
\hline \multicolumn{3}{|l|}{ Chemotherapy } \\
\hline Yes & 0 & 0 \\
\hline No & 5.9 & 5.9 \\
\hline \multicolumn{3}{|c|}{$\begin{array}{l}\text { Number of metastatic } \\
\text { organs except bone }\end{array}$} \\
\hline 0 & 0 & 0 \\
\hline 1 & 4.4 & 4.7 \\
\hline$\geq 2$ & 8.7 & 9.4 \\
\hline
\end{tabular}

Grade I: well differentiated; Grade II: moderately differentiated; Grade III: poorly differentiated; Grade IV: undifferentiated anaplastic. OS, overall survival; CSS, cancer-specific survival.

nomogram prediction has an obvious correlation with actual observation. The $\mathrm{C}$-indices of $\mathrm{OS}$ in training and validation cohorts were 0.705 (95\% CI, 0.691-0.719) and 0.678 (95\%
CI, 0.661-0.695), respectively. The C-indices of CSS in training and validation cohorts were 0.710 (95\% CI, $0.696-$ 0.724 ) and 0.684 (95\% CI, 0.666-0.702), respectively.

\section{Discussion}

BC patients with bone metastasis were more likely to experience poor prognosis and quality of life $(9,10)$. Moreover, little is known about how to accurately predict the prognosis of this population. In order to provide some useful insights into the prognosis and treatment strategies for this challenging disease, we retrospectively analyzed BC patients with bone metastasis at presentation $(n=5,860)$ from the SEER database.

In this study, we identified seven independent prognostic predictors of BC patients with bone metastasis. Tumor grade was a significant variable of OS and CSS, which was in accordance with the previous studies $(31,32)$. Histological type was found to be an independent predictor of survival among $\mathrm{BC}$ patients with bone metastasis. Patients in ductal group had better survival compared with lobular group. Tumor size is usually recognized as an important risk factor of survival among BC patients $(33,34)$. Our multivariate analysis also revealed that tumor size less than $5 \mathrm{~cm}$ was significantly associated with increased survival. Kim et al. (35) reported that molecular subtype can predict the prognosis for BC patients with brain metastasis. The TNBC subtype as an aggressive form, showed the worst prognosis in $\mathrm{BC}$ patients with brain metastasis, consistent with our results. Moreover, patients with bone-only metastasis had better survival than that of patients with additional visceral metastasis. Other studies also reached the same conclusion $(6,31,36)$. One possible reason for this may be that bone is not a vital organ (31).

Surgical treatment for primary lesion is generally performed as a palliative surgery for metastatic BC patients. Recently, some studies reported that local surgery may achieve improvement in survival of metastatic BC (37-40). Moreover, Xiong et al. (40) reported that surgical treatment for primary lesion prolonged survival among selected stage IV BC patients, such as those with bone- or soft tissueonly metastasis. Similarly, our multivariate analysis showed that local surgery significantly improved the survival. For metastatic BC, chemotherapy is recommended as it can prolong survival, decrease cancer-related complications, and improve quality of life (41). Our research also revealed that $\mathrm{BC}$ patients with bone metastasis who received 

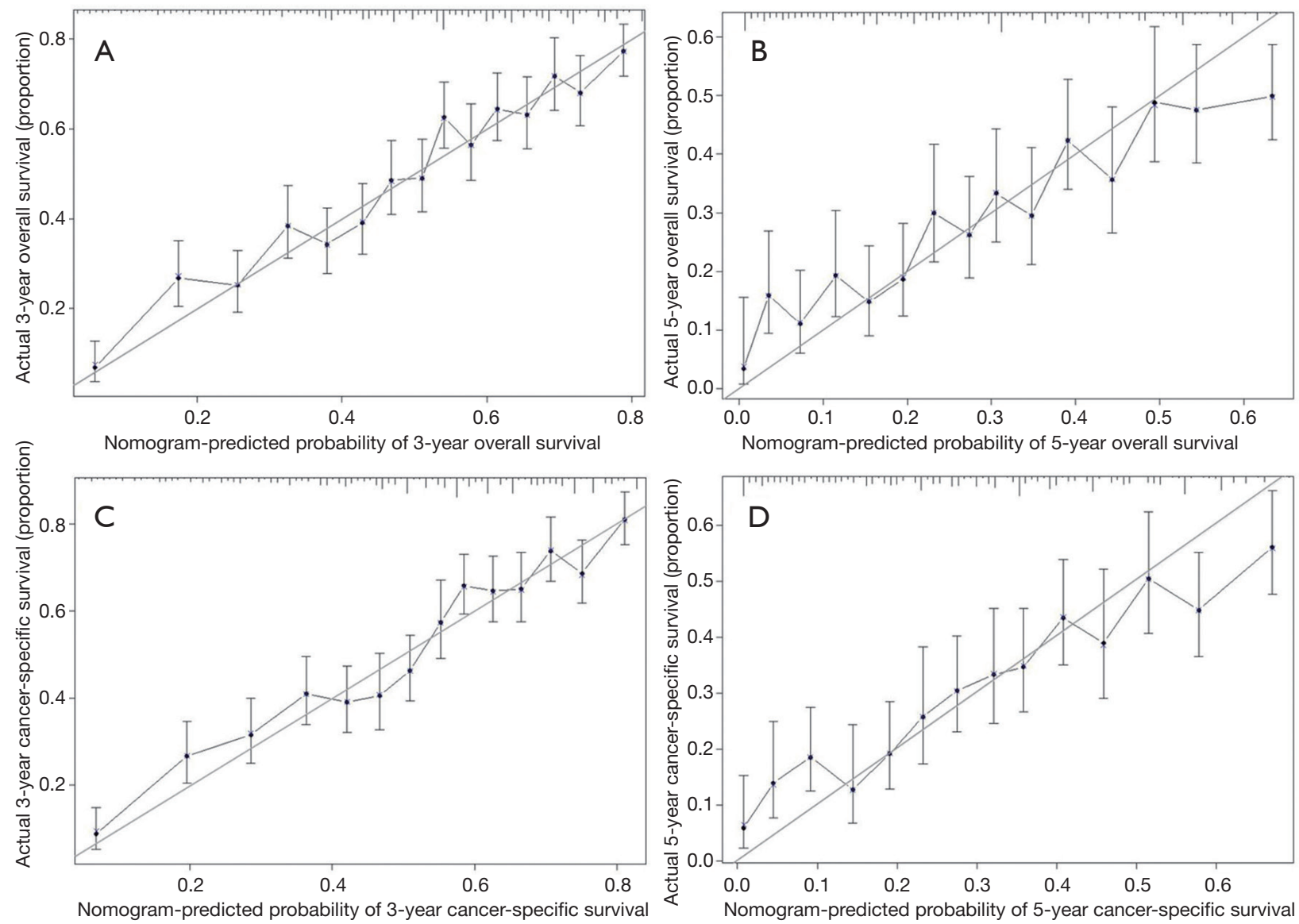

Figure 4 Calibration curves of the nomogram in the training cohort. Prediction of 3- (A) and 5-year (B) overall survival; and prediction of 3(C) and 5-year (D) cancer-specific survival.

chemotherapy can achieve survival benefits. It is generally accepted that radiotherapy has the potential to alleviate pain and achieve good local control. Some studies reported that breast radiotherapy is associated with improved survival in metastatic patients $(42,43)$. However, our multivariate analysis failed to identify radiotherapy as a significant predictor of either OS or CSS. Roayaei et al. (44) also supported this finding and showed no effect of radiotherapy of the breast on survival in metastatic disease.

In order to maximize prognostic ability, we established the nomograms (Figures 2,3) based on these independent variables. Our developed nomograms presented adequate discriminatory ability and obvious correlation between prediction and actual observation. Clinicians can refer to our nomograms to recommend appropriate treatment for BC patients with bone metastasis. Nomograms are widely constructed for predicting the outcome of other different
$\mathrm{BC}$ populations and showed advantage in their management (45-47). Additionally, Yang et al. (48) established a userfriendly nomogram to preoperatively predict axillary lymph node metastasis, which is helpful to optimize treatment methods of BC patients. Delpech et al. (49) developed a clinical nomogram for probability prediction of bone-only metastasis among non-metastatic BC patients based on five independent predictors.

Several limitations in this research should be acknowledged. One limitation was that this was a retrospective study, which may generate inevitable biases. Second, the SEER database does not contain data about recurrence or specific treatment, which may affect the clinical outcome. The third limitation was that other important factors such as specific site of bone metastasis, treatment for bone metastasis, were not included in the database. Those important variables should be considered in the future research. 

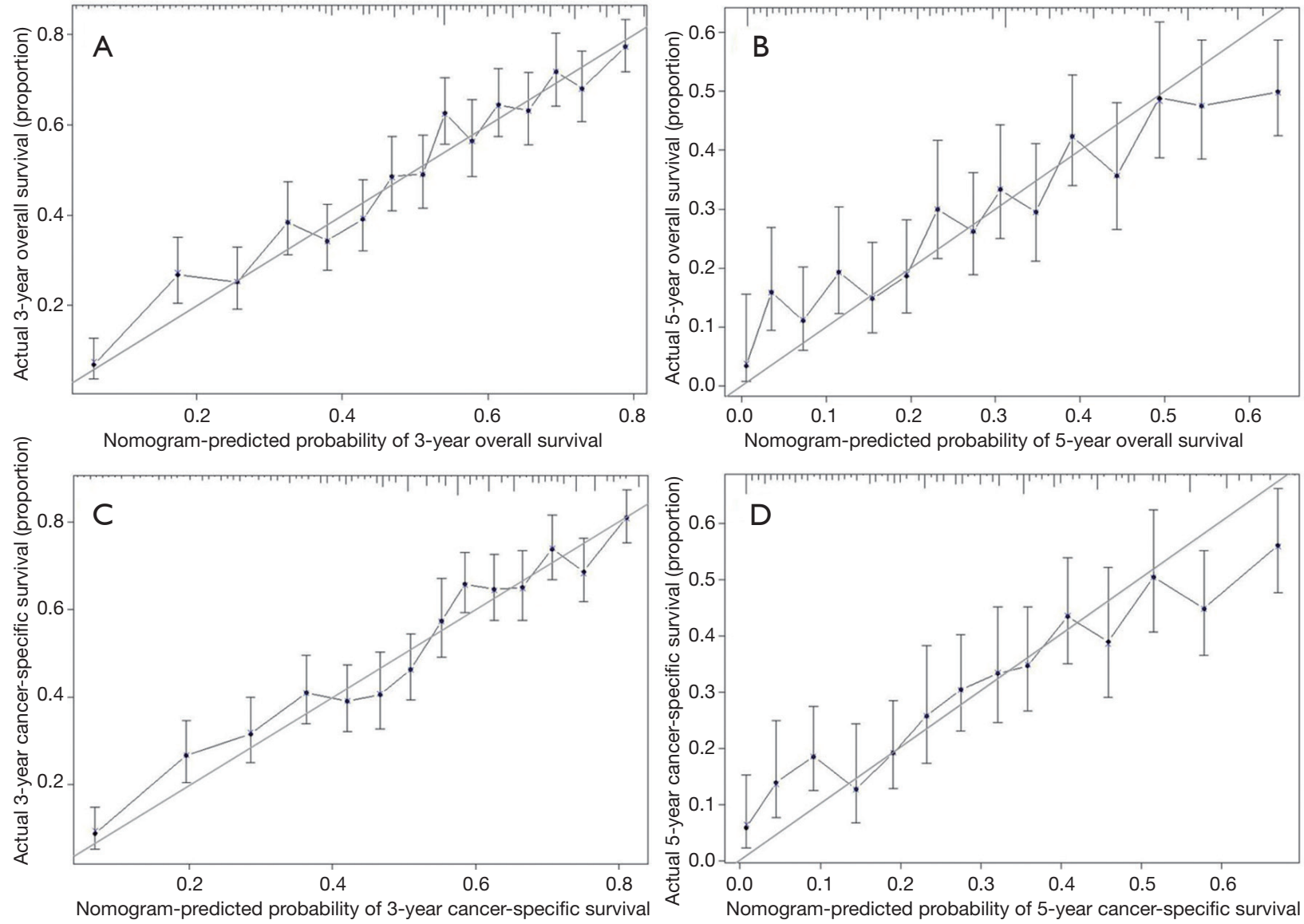

Figure 5 Calibration curves of the nomogram in the validation cohort. Prediction of 3- (A) and 5-year (B) overall survival; and prediction of 3(C) and 5-year (D) cancer-specific survival.

\section{Conclusions}

Our novel prognostic nomograms for BC patients with bone metastasis can provide more accurate survival information for clinicians and facilitate them to provide appropriate treatment measures for metastatic lesions. Meanwhile, we propose more external validation to further refine our conclusions.

\section{Acknowledgments}

Funding: This work was supported by the grants from National Key R\&D Program of China (2018YFC1105400), National Natural Science Foundation of China (81702118), 2018 Zhejiang University Academic Award for Outstanding Doctoral Candidates and Medical and Health Science and Technology Plan of Department of Health of Zhejiang Province (WKJ-ZJ-1821).

\section{Footnote}

Conflicts of Interest: The authors have no conflicts of interest to declare.

Ethical Statement: The authors are accountable for all aspects of the work in ensuring that questions related to the accuracy or integrity of any part of the work are appropriately investigated and resolved. This study was undertaken in accordance of standard guidelines and the Declaration of Helsinki and approved by the Ethics Committee of the Second Affiliated Hospital of Zhejiang University School of Medicine.

Open Access Statement: This is an Open Access article distributed in accordance with the Creative Commons Attribution-NonCommercial-NoDerivs 4.0 International License (CC BY-NC-ND 4.0), which permits the non- 
commercial replication and distribution of the article with the strict proviso that no changes or edits are made and the original work is properly cited (including links to both the formal publication through the relevant DOI and the license). See: https://creativecommons.org/licenses/by-nc-nd/4.0/.

\section{References}

1. Roodman GD. Mechanisms of bone metastasis. N Engl J Med 2004;350:1655-64.

2. Monne M, Piras G, Fancello P, et al. Identification of a founder BRCA2 mutation in Sardinian breast cancer families. Fam Cancer 2007;6:73-9.

3. Kuchuk I, Hutton B, Moretto P, et al. Incidence, consequences and treatment of bone metastases in breast cancer patients-Experience from a single cancer centre. J Bone Oncol 2013;2:137-44.

4. Sherry MM, Greco FA, Johnson DH, et al. Metastatic breast cancer confined to the skeletal system. An indolent disease. Am J Med 1986;81:381-6.

5. Scheid V, Buzdar AU, Smith TL, et al. Clinical course of breast cancer patients with osseous metastasis treated with combination chemotherapy. Cancer 1986;58:2589-93.

6. Plunkett TA, Smith P, Rubens RD. Risk of complications from bone metastases in breast cancer. implications for management. Eur J Cancer 2000;36:476-82.

7. Kono M, Fujii T, Matsuda N, et al. Somatic mutations, clinicopathologic characteristics, and survival in patients with untreated breast cancer with bone-only and non-bone sites of first metastasis. J Cancer 2018;9:3640-6.

8. Coleman RE. Skeletal complications of malignancy. Cancer 1997;80:1588-94.

9. Costa L, Badia X, Chow E, et al. Impact of skeletal complications on patients' quality of life, mobility, and functional independence. Support Care Cancer 2008;16:879-89.

10. Brook N, Brook E, Dharmarajan A, et al. Breast cancer bone metastases: pathogenesis and therapeutic targets. Int J Biochem Cell Biol 2018;96:63-78.

11. Hortobagyi GN, Theriault RL, Porter L, et al. Efficacy of pamidronate in reducing skeletal complications in patients with breast cancer and lytic bone metastases. Protocol 19 Aredia Breast Cancer Study Group. N Engl J Med 1996;335:1785-91.

12. Svensson E, Christiansen CF, Ulrichsen SP, et al. Survival after bone metastasis by primary cancer type: a Danish population-based cohort study. BMJ open 2017;7:e016022.

13. Pogoda K, Niwinska A, Murawska M, et al. Analysis of pattern, time and risk factors influencing recurrence in triple-negative breast cancer patients. Med Oncol 2013;30:388.

14. Finn RS, Crown JP, Lang I, et al. The cyclin-dependent kinase 4/6 inhibitor palbociclib in combination with letrozole versus letrozole alone as first-line treatment of oestrogen receptor-positive, HER2-negative, advanced breast cancer (PALOMA-1/TRIO-18): a randomised phase 2 study. Lancet Oncol 2015;16:25-35.

15. Swain SM, Baselga J, Kim SB, et al. Pertuzumab, trastuzumab, and docetaxel in HER2-positive metastatic breast cancer. N Engl J Med 2015;372:724-34.

16. Nathan SS, Healey JH, Mellano D, et al. Survival in patients operated on for pathologic fracture: implications for end-of-life orthopedic care. J Clin Oncol 2005;23:6072-82.

17. Ratasvuori M, Wedin R, Hansen BH, et al. Prognostic role of en-bloc resection and late onset of bone metastasis in patients with bone-seeking carcinomas of the kidney, breast, lung, and prostate: SSG study on 672 operated skeletal metastases. J Surg Oncol 2014;110:360-5.

18. Guzik G. Oncological and functional results after surgical treatment of bone metastases at the proximal femur. BMC Surg 2018;18:5.

19. Adli M, Kuzhan A, Alkis H, et al. FDG PET uptake as a predictor of pain response in palliative radiation therapy in patients with bone metastasis. Radiology 2013;269:850-6.

20. Christensen MH, Petersen LJ. Radionuclide treatment of painful bone metastases in patients with breast cancer: a systematic review. Cancer Treat Rev 2012;38:164-71.

21. Ahn SG, Lee HM, Cho SH, et al. Prognostic factors for patients with bone-only metastasis in breast cancer. Yonsei Med J 2013;54:1168-77.

22. Parkes A, Warneke CL, Clifton K, et al. Prognostic Factors in Patients with Metastatic Breast Cancer with Bone-Only Metastases. Oncologist 2018;23:1282-8.

23. Zhao C, Zhang Z, Zhong N, et al. Outcomes and prognostic factors for surgically treated patients with breast cancer spine metastases. J Bone Oncol 2018;12:38-43.

24. Xiong Z, Deng G, Huang X, et al. Bone metastasis pattern in initial metastatic breast cancer: a population-based study. Cancer Manag Res 2018;10:287-95.

25. Zhang Z, Jiang H, Chen J, et al. Hepatocellular carcinoma: radiomics nomogram on gadoxetic acid-enhanced MR imaging for early postoperative recurrence prediction. Cancer Imaging 2019;19:22.

26. Gross JP, Whelan TJ, Parulekar WR, et al. Development and validation of a nomogram to predict lymphedema following axillary surgery and radiotherapy in women with 
breast cancer from the NCIC CTG MA.20 randomized trial. Int J Radiat Oncol Biol Phys 2019; 105:165-73.

27. Yan P, Huang R, Hu P, et al. Nomograms for predicting the overall and cause-specific survival in patients with malignant peripheral nerve sheath tumor: a populationbased study. J Neurooncol 2019; 143:495-503.

28. Wang Z, Li S, Li Y, et al. Prognostic factors for survival among patients with primary bone sarcomas of small bones. Cancer Manag Res 2018;10:1191-9.

29. Xue M, Chen G, Dai J, et al. Development and Validation of a Prognostic Nomogram for Extremity Soft Tissue Leiomyosarcoma. Front Oncol 2019;9:346.

30. Song $W$, Zhu ZG, Wu Q, et al. A nomogram to predict overall survival for biliary tract cancer. Cancer Manag Res 2018;10:1535-41.

31. Gong Y, Zhang J, Ji P, et al. Incidence proportions and prognosis of breast cancer patients with bone metastases at initial diagnosis. Cancer Med 2018;7:4156-69.

32. Houvenaeghel G, Goncalves A, Classe JM, et al. Characteristics and clinical outcome of T1 breast cancer: a multicenter retrospective cohort study. Ann Oncol 2014;25:623-8.

33. Michaelson JS, Silverstein M, Wyatt J, et al. Predicting the survival of patients with breast carcinoma using tumor size. Cancer 2002;95:713-23.

34. Cortesi L, Marcheselli L, Guarneri V, et al. Tumor size, node status, grading, HER2 and estrogen receptor status still retain a strong value in patients with operable breast cancer diagnosed in recent years. Int J Cancer 2013;132:E58-65.

35. Kim YJ, Kim JS, Kim IA. Molecular subtype predicts incidence and prognosis of brain metastasis from breast cancer in SEER database. J Cancer Res Clin Oncol 2018;144:1803-16.

36. Jacobson AF, Shapiro CL, Van den Abbeele AD, et al. Prognostic significance of the number of bone scan abnormalities at the time of initial bone metastatic recurrence in breast carcinoma. Cancer 2001;91:17-24.

37. Akay CL, Ueno NT, Chisholm GB, et al. Primary tumor resection as a component of multimodality treatment may improve local control and survival in patients with stage IV inflammatory breast cancer. Cancer 2014;120:1319-28.

38. Tan $\mathrm{Y}, \mathrm{Li} X$, Chen H, et al. Hormone receptor status may impact the survival benefit of surgery in stage iv breast cancer: a population-based study. Oncotarget 2016;7:70991-1000.

39. Rashaan ZM, Bastiaannet E, Portielje JE, et al. Surgery in metastatic breast cancer: patients with a favorable profile seem to have the most benefit from surgery. Eur J Surg Oncol 2012;38:52-6.

40. Xiong Z, Deng G, Wang J, et al. Could local surgery improve survival in de novo stage IV breast cancer? BMC Cancer 2018;18:885.

41. Mehta RS, Barlow WE, Albain KS, et al. Overall Survival with Fulvestrant plus Anastrozole in Metastatic Breast Cancer. N Engl J Med 2019;380:1226-34.

42. Le Scodan R, Stevens D, Brain E, et al. Breast cancer with synchronous metastases: survival impact of exclusive locoregional radiotherapy. J Clin Oncol 2009;27:1375-81.

43. Choi SH, Kim JW, Choi J, et al. Locoregional Treatment of the Primary Tumor in Patients With De Novo Stage IV Breast Cancer: A Radiation Oncologist's Perspective. Clin Breast Cancer 2018;18:e167-e178.

44. Roayaei M, Nikanpour Y, Mahdavi H. Effect of Surgery or Radiotherapy of the Breast on Survival in Metastatic Disease: a Retrospective Cohort Study. Indian J Surg Oncol 2019;10:213-8.

45. Sun W, Cheng M, Zhou H, et al. Nomogram Predicting Cause-Specific Mortality in Nonmetastatic Male Breast Cancer: A Competing Risk Analysis. J Cancer 2019;10:583-93.

46. Wang K, Li HL, Xiong YF, et al. Development and validation of nomograms integrating immune-related genomic signatures with clinicopathologic features to improve prognosis and predictive value of triple-negative breast cancer: A gene expression-based retrospective study. Cancer Med 2019;8:686-700.

47. Zhao S, Shen W, Du R, et al. Three inflammation-related genes could predict risk in prognosis and metastasis of patients with breast cancer. Cancer Med 2019;8:593-605.

48. Yang J, Wang T, Yang L, et al. Preoperative Prediction of Axillary Lymph Node Metastasis in Breast Cancer Using Mammography-Based Radiomics Method. Sci Rep 2019;9:4429.

49. Delpech Y, Bashour SI, Lousquy R, et al. Clinical nomogram to predict bone-only metastasis in patients with early breast carcinoma. Br J Cancer 2015;113:1003-9.

Cite this article as: Wang Z, Cheng Y, Chen S, Shao H, Chen X, Wang Z, Wang Y, Zhou H, Chen T, Lin N, Ye Z. Novel prognostic nomograms for female patients with breast cancer and bone metastasis at presentation. Ann Transl Med 2020;8(5):197. doi: 10.21037/atm.2020.01.37 